the irregular change of electrocardiogram in the patients with AMI should be necessary in diagnosis and therapy.

Methods The retrospective study enrolled 10 subjects with AMI who were hospitalisation in Cangzhou Central Hospital from May 2009 to May 2011. The changes of ECG were irregular in all of them. ECG, enzymes (due to myocardiolysis) and echocardiography were detected, percutaneous coronary intervention (PCI) was given also.

Results 10 patients with AMI included four male, six female, aged $32-72(57 \pm 4.16) ; 1 \pm 0.18 \mathrm{~h}$ were continuing from chest pain seizures to hospitalisation; No-dynamic change in ECGs in acute phase; Troponin was increased in two cases; serum creatine kinase and troponin I both increased in eight cases; completely morbid change of leftcircumflex in six cases and capital morbid change of leftcircumflex in four cases were detected by coronary angiography.

Conclusions The atypical change of electrocardiogram in patients with acute myocardial infarction mostly predicts that infarct related artery is leftcircumflex.

[gw22-e0626] STUDY ON ATYPICAL CHANGE OF

\title{
ELECTROCARDIOGRAM IN PATIENTS WITH ACUTE MYOCARDIAL INFARCTION
}

Zhang Jun, Wang Chang-Hou, Yuan Chen, Fu Jin-Guo, Niu He-Ping, Wan Yan-Fang, Fei Ling, Wang Jun, Chen Shu-Ping Department of Cardiology, Cangzhou Central Hospital, The Clinical College of Hebei Medical University, Cangzhou, China

10.1136/heartjnl-2011-300867.471

Purpose The role of electrocardiogram (ECG) in the diagnosis of acute myocardial infarction (AMI) is very important. As an emergency technique, electrocardiogram is easy to operate, although, to diagnose acute myocardial infarction (AMI) by electrocardiogram become difficult due to the irregular change of electrocardiogram in the patients with AMI. Diagnostic errors, missed diagnosis even delay therapy appeared. To assess 